\title{
Estimating modes of a complex dynamical network from impulse response data: Structural and graph-theoretic characterizations
}

\author{
Yan Wan ${ }^{1, *, \dagger}$, Sandip Roy ${ }^{2}$, Mengran $\mathrm{Xue}^{3}$ and Veenadhar Katragadda ${ }^{1}$ \\ ${ }^{1}$ Department of Electrical Engineering, University of North Texas, Denton, TX 76207, USA \\ ${ }^{2}$ School of Computer Science and Engineering, Washington State University, Pullman, WA 99163, USA \\ ${ }^{3}$ Electrical Engineering and Computer Science, University of Michigan, Ann Arbor, MI 48109, USA
}

\begin{abstract}
SUMMARY
We examine the role played by a linear dynamical network's topology in inference of its eigenvalues from noisy impulse-response data. Specifically, for a canonical linear-time-invariant network dynamics, we relate the Cramer-Rao bounds on eigenvalue estimator performance (from impulse-response data) to structural properties of the transfer function and in turn, to the network's topological structure. We begin by reviewing and enhancing algebraic characterizations of such eigenvalue estimates, which are based on pole-residue and pole-zero representations of the network's dynamics. We use these results to characterize mode estimation in networks with slow-coherency structures, finding that stimulus and observation in each strongly connected network subgraph is needed for high-fidelity estimation. We also obtain spectral and graphical characterizations of estimator performance for other graph classes (e.g., trees) and for the general case. These characterizations are used to determine the role of measurement and actuation locations in estimation performance. Finally, application of our results in dynamical-network security is illustrated through a simple example, and a concrete procedure for network mode estimation that draws on our structural results is introduced to conclude the article. Copyright $\odot 2014$ John Wiley \& Sons, Ltd.
\end{abstract}

Received 21 February 2013; Revised 18 December 2013; Accepted 9 January 2014

KEY WORDS: mode estimation; network topology; linear response; dynamical networks

\section{INTRODUCTION}

The inference of system parameters from measurement data, or through deliberate stimulation and measurement, has been extensively studied in both the signal processing and controls communities under the various headings of system identification, parameter estimation, and deconvolution (e.g., [1-3]). Recently, the problem of inferring network dynamics and models from observations has come to the forefront, in such diverse fields as electric power-system control, biological signaling network analysis, and security analysis of cyber-physical networks [4-7]. Several network parameter estimation problems have the following general form: a detailed model for the network interactions is not known, but some information about the network's topology is available (e.g., aggregate statistics like average node degree, an illustration of the network graph, or knowledge the graph is of a certain type). Inferring the detailed model of the network dynamics, or important statistics thereof (e.g., modes or eigenvalues or time-constants, critical interaction parameters, or unknown network-wide statistics), is the primary goal. Information about the underlying network topology can allow improved estimation, by (i) giving insight into the observation and stimulation locations that best permit inference from noisy data and (ii) constraining the possible dynamics of the system. The purpose of this article is to explore the role played by a network's

\footnotetext{
*Correspondence to: Yan Wan, Electrical Engineering, University of North Texas, Denton, TX 76207, USA.

†E-mail: yan.wan@unt.edu
} 
topological structure in estimation of the network's time constants and other parameters from noisy data (see also [8]). Specifically, we focus on developing network-theoretic bounds on estimator performance, so as to evaluate where stimuli and measurements should be placed in the network for accurate estimation.

Within the broad literature on parameter estimation [1-3], our studies here are most closely related to estimation methods for auto-regressive moving average models (or, equivalently, discretetime SISO linear-time-invariant system models) from noisy impulse-response data [3]. Within this domain, several results are concerned with estimating nonrandom parameters in the pole-zero and/or pole-residue representation of the model, or on finding Cramer-Rao bounds (CRBs) on the estimates, for example, [3, 9]. Here, we also consider the problem of estimating a discrete-time linear system's modes from impulse-response data. The contribution of this work lies in connecting the estimation performance for a network model to its graph structure. Specifically, we make explicit the dependence of the Cramer-Rao bounds, which lower bound the best possible performance of mode estimates, on the pole-structure and zero structure of the transfer function. In turn, we use these relationships to characterize the Cramer-Rao bounds in terms of an underlying network structure imposed on the system dynamics.

Our effort here is also closely related to the literature on synchronization and diffusion processes in networks (e.g., [10-12]), which establishes relationships between graph structures and the modes of the network's dynamics. For instance, in [12], the relationship between node degrees in a multi-layer graph and the second-largest eigenvalue of the associated consensus (synchronization) dynamics was established. In this paper, the performance of network mode estimation from sparse observations is being considered and tied to the graph structure. As complex performance measures are being analyzed (e.g., trace or determinant of the Cramer-Rao-bound matrix), the graph-theoretic and structural results are different from classical analyses of the dynamics themselves, however, our analyses draw on similar algebraic-graph-theory constructs.

The inference problem that we address here is motivated by network estimation problems in infrastructure management, biological network inference, and sensor/vehicle networking. Among these applications, inference problems have been particularly well-studied for biological systems, wherein the characterization of biochemical/bioelectrical processes from noisy and incomplete measurements of transients is needed (e.g., [4]). Of interest, numerous works have applied system identification and statistical parameter estimation techniques to infer various biological systems' dynamics, including cell-signaling and neuronal-network dynamics. These methods do not, however, specifically exploit the topological structure of the network. Recently, the need for characterizing the role of the network topology in inference, and in turn developing inference techniques that exploit the topological structure, has been recognized. In a complementary direction, static and stationary interactions in biological networks (rather than network dynamics) have been identified from experimental data using machine-learning techniques (e.g., [5]). Recently, these efforts have been generalized toward the inference of dynamic Bayesian networks [5]; however, the performance of the heuristic algorithms used has not been tied to the network's topological structure. In contrast to the biological networks, the literature on engineered networks has largely focused on control and design rather than network inference (e.g., [13]). However, several recent works have commented on the need for inferring structural and dynamic characteristics of these large-scale networks, and network mode estimation has been explicitly studied in the context of electric power-system analysis (e.g., [14]). In particular, motivated by the need for estimation/identification from phasor measurement unit data and other observations, power engineers have sought for algorithms for mode and mode-shape analysis from observations $[15,16]$. Recently, the estimation of linear network dynamics and models from sparse local measurements has also been pursued as part of an initiative on security/vulnerability analysis of cyber-physical systems [6, 17]. Our efforts here enhance these results by providing system-structural and graph-theoretic bounds on mode estimator performance, in cases where only localized stimulation and measurement are possible.

\footnotetext{
${ }^{\ddagger}$ It is worth stressing that there is a significant literature on system identification upon persistent rather than impulsive stimulation. We focus here on identification from impulse-response data because the network applications that we consider typically do not permit deliberate persistent stimulation.
} 
The remainder of the article is organized as follows. In Section 2, we formulate the canonical network mode (eigenvalue) estimation problem. Section 3 describes our core results, on systemstructure-based and network-structure-based Cramer-Rao bounds of the network dynamics' modes. Many of our results apply to general network topologies, but we also obtain keener bounds for networks with slow-coherency structures [18] and tree graphs and obtain results on measurement and actuation placement. Section 4 illustrates the structural results on estimation performance in two examples, including one focused on dynamical-network security. Planned future work is briefly discussed in Section 5.

\section{FORMULATION: MODE INFERENCE FOR A NETWORK DIFFUSION PROCESS}

Mode estimation in a canonical model for diffusive network dynamics is considered here [19], which arises in such diverse domains as synchronization in biological systems, autonomous-vehicle control, and analysis of power-system transients [20]. We focus on a discrete-time model because inference is typically performed using a recorded (discrete) data set and also to maintain consistency with the literature on auto-regressive moving average model estimation [3].

The network dynamics are defined on a weighted graph. Formally, an undirected and weighted graph $\Gamma$ with $n$ vertices, labeled $1, \ldots, n$, is considered. Edges are specified by pairs of distinct vertices, and each edge has associated with it a real scalar weight. In a departure from the standard graph theory, we shall (in some parts of this paper) allow the edge weights in the graph $\Gamma$ to be negative.

The diffusion dynamics of interest are defined from the Laplacian matrix of the graph. Formally, the $n \times n$ Laplacian matrix $L(\Gamma)$ is defined from the graph $\Gamma$ in the standard way, as follows. The entry $L_{i j}$ at row $i$ and column $j, i \neq j$, is set equal to negative of the edge weight between vertices $i$ and $j$ if there is an edge between them and set to zero if there is not. Additionally, the matrix's diagonal entries are set so that each row sums to zero, that is, as $L_{i i}=-\sum_{j \neq i} L_{i j}$, for $i=1, \ldots, n$.

The network model tracks the evolution of real scalar states $x_{i}[k]$ associated with each component $i=1, \ldots, n$ in a network, in discrete time $\left(k \in Z^{+}\right)$. The components' states evolve through diffusion among the network components (according to the graph $\Gamma$ ), as well as through the stimulus of the network at a single component $i$. In particular, the full state $\mathbf{x}[k]=\left[x_{1}[k] \ldots x_{n}[k]\right]^{T}$ evolves according to the difference equation

$$
\mathbf{x}[k+1]=\mathbf{x}[k]-L \mathbf{x}[k]+\mathbf{e}_{i} u[k],
$$

where ${ }^{T}$ denotes the transpose, $\mathbf{e}_{i}$ is a $0-1$ indicator vector with $i$ th entry equal to 1 , and $u[k]$ is a scalar input signal that represents a stimulus at component $i$. We assume an impulse input, that is, $u[k]=1$ for $k=0$ and $u[k]=0$ otherwise, and a nil initial state. We also assume that noisy observations $y[k]$ are made of one component $j$ 's state:

$$
y[k]=\mathbf{e}_{j}^{T} \mathbf{x}[k]+w[k],
$$

where $w[k]$ is a discrete-time stationary zero-mean Gaussian white noise process with variance $\sigma^{2}=1$ Without Loss of Generality (WLOG). We refer to the aforementioned model, specified in (1) and (2), as the Laplacian network.

Several properties of the Laplacian network's dynamics and specifically its spectrum are worth highlighting. First, we note that the observed dynamics are entirely specified by the graph $\Gamma$ and the locations of the stimulus and the observation (measurement). The state matrix of the network dynamics - which depends solely on the graph $\Gamma$-is $A=I_{n}-L$, where $I_{n}$ is an $n \times n$ identity matrix. Several characterizations of the spectrum (eigenvalues and eigenvectors) of the state matrix $A$ follow immediately from well-known spectral properties of Laplacian matrices. First, the eigenvalues of the state matrix $A$ are all real and at least one eigenvalue equals 1. Furthermore, in the case where the edge weights are known to be nonnegative, the eigenvalues are all less than or equal to 1 , with the number of unity eigenvalues equal to the number of disconnected subgraphs of $\Gamma$. We note that the eigenvalues may or may not be within the unit circle, and so the dynamics may be stable or 
unstable. In a range of applications (for instance, when a continuous-time dynamics are sampled), the edge weights are sufficiently small that the state matrix's eigenvalues are in the unit circle and the dynamics are stable. We will focus on this typical case in our later analysis.

Let us now formalize the network mode identification problem, beginning with assumptions about what is known a priori to the estimator about the network dynamics. Firstly, we assume that the user knows the dimension of the network. This is often the case in real-world problems, either because the network is human-engineered or because the identification follows on other preliminary experimentation. For instance, prior to detailed construction of a genetic network, the genes involved in a process are determined, which specifies the network dimension. Secondly, we assume that the user either can choose or knows the stimulus and observation locations-most of our results assume this knowledge. For instance, in structural-engineering and many infrastructure-network applications, disturbance sources are known, and the disturbance impact is observed at other locations. Thirdly, we also may have some information about the structure of the network graph: the graph may be known to belong to a particular class (e.g., a random graph), some aggregate properties of the graph may be known, or (an illustration of) the map (graph) may be available; this is especially common in many infrastructure-type applications. Critically, the graph's edge weights and hence the modes are not known and are modeled as unknown nonrandom parameters. Disturbance excitations may be known to impact certain locations in the network (or impulse inputs may be deliberately applied), and local observations of the impact can be used to identify the network dynamics. Hence, identification from impulse-response data is of importance.

Our focus here is on identifying the modes (eigenvalues) of the matrix $L$ or equivalently of the state matrix $A$ from the impulse response $y[k]$. These modes are crucial measures of the dynamics, in that they capture settling rates and other transient characteristics. Because the network dynamics are diffusive, it is typical that the state matrix will be known to have a unity eigenvalue, and the estimation goal is to determine the remaining $n-1$ eigenvalues. In fact, mode estimation for linear systems from impulse response data has been extensively studied [3, 9]. Our focus will be on relating bounds on the best possible estimation performance to the graph $\Gamma$ and the observation and measurement locations, with the view of informing experiment design and better understanding of how the graph and stimulation/observation structure modulate estimability. Noting that the mode estimation problem is a nonrandom parameter estimation problem, a lower bound on the estimation error variance is given by the Cramer-Rao bound matrix. Our focus here will be on developing graphical characterizations of the Cramer-Rao bound matrix and metrics defined thereof.

\section{STRUCTURAL AND GRAPH-THEORETIC CHARACTERIZATIONS OF CRAMER-RAO BOUNDS IN MODE ESTIMATION}

Maximum likelihood (ML) estimation of a single-input-single-output LTI system's modes from noisy impulse-response data has been studied, and explicit calculations of the Cramer-Rao bound have been obtained using the pole-residue form of the system's transfer function [9]. Our focus here is to derive further performance bounds in terms of the pole-zero structure and eigenstructure of the system. In turn, we use this structural characterization to give graph-theoretic bounds on estimator performance, for the Laplacian network.

\subsection{Relationship between poles/residues and Cramer-Rao bounds}

We express the Cramer-Rao bounds on Laplacian-network mode estimates in terms of the system's poles and residues (closely following the analysis of [3]) and draw on these bounds to develop simple relationships between pole and residue locations and estimation error.

Because the state matrix $A$ of the Laplacian network is symmetric, it has only real, simple eigenvalues. The transfer function $H(z)$ for the Laplacian network can thus be written in the following pole-residue form:

$$
H(z)=\sum_{i=0}^{n-1} \frac{A_{i}}{z-p_{i}},
$$


where $p_{0} \geqslant p_{1} \geqslant \ldots \geqslant p_{n-1}$ are the poles (or modes or eigenvalues) of the system, $p_{0}$ is unity, and $A_{0}, \ldots, A_{n-1}$ are the corresponding residues. We note that the pole-residue representation of the transfer function is unique in the case that the poles are nonrepeated. It is also worth stressing that the residues $A_{i}$ depend on both the network graph and the stimulus/observation locations, while the poles depend only on the network graph as described earlier.

We develop the Cramer-Rao bound in the case where the modes are distinct, because the dynamics are unobservable in the case of indistinct models. Because we are interested in the role of network structure in the Cramer-Rao bounds and not in the limitations imposed by insufficient data, we largely focus on the limiting case of an infinite measurement horizon (i.e., the infinite

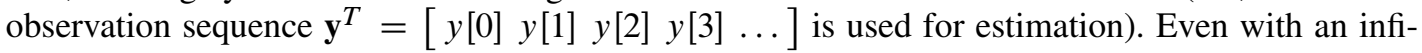
nite observation horizon, all eigenvalues except the unity eigenvalue cannot be estimated perfectly because the corresponding modal responses have finite energy.

We now derive algebraic expressions for the Cramer-Rao bounds on the pole and residue estimates. For this problem, the Fisher information matrix can be calculated as

$$
F(\theta)=\frac{\partial \mathbf{x}_{j}^{T}}{\partial \theta} \frac{\partial \mathbf{x}_{j}}{\partial \theta}=\left[\begin{array}{c}
\frac{\partial \mathbf{x}_{j}^{T}}{\partial \mathbf{A}} \\
\frac{\partial \mathbf{x}_{j}^{T}}{\partial \mathbf{p}}
\end{array}\right]\left[\begin{array}{ll}
\frac{\partial \mathbf{x}_{j}}{\partial \mathbf{A}} & \frac{\partial \mathbf{x}_{j}}{\partial \mathbf{p}}
\end{array}\right],
$$

see [9]. Here $\theta=[\mathbf{A} \mathbf{p}]$ is the parameter vector, where $\mathbf{A}=\left[\begin{array}{ll}A_{0} A_{1} \ldots A_{n-1}\end{array}\right], \mathbf{p}=$ $\left[\begin{array}{llll}p_{0} & p_{1} & \ldots & p_{n-1}\end{array}\right]$ and $\mathbf{x}_{j}^{T}=\left[x_{j}[0] x_{j}[1] x_{j}[2] x_{j}[3] \ldots\right]$ is the noiseless impulse response of the system (1). Now let us express $F(\theta)$ in terms of the system parameters $\theta$. From the transfer function $H(z)$, we obtain that $x_{j}[k]=\sum_{i=0}^{n-1} A_{i} p_{i}^{k}$, and hence

$$
\frac{\partial \mathbf{x}_{j}^{T}}{\partial \mathbf{A}}=\left[\begin{array}{ccccc}
1 & p_{0} & p_{0}^{2} & p_{0}^{3} & \cdots \\
1 & p_{1} & p_{1}^{2} & p_{1}^{3} & \cdots \\
\vdots & \vdots & \vdots & \vdots & \vdots \\
1 & p_{n-1} & p_{n-1}^{2} & p_{n-1}^{3} & \cdots
\end{array}\right] \text { and } \frac{\partial \mathbf{x}_{j}^{T}}{\partial \mathbf{p}}=\left[\begin{array}{ccccc}
0 & A_{0} & 2 A_{0} p_{0} & 3 A_{0} p_{0}^{2} & \cdots \\
0 & A_{1} & 2 A_{1} p_{1} & 3 A_{1} p_{1}^{2} & \ldots \\
\vdots & \vdots & \vdots & \vdots & \vdots \\
0 & A_{n-1} & 2 A_{n-1} p_{n-1} & 3 A_{n-1} p_{n-1}^{2} & \cdots
\end{array}\right] \text {. }
$$

The Cramer-Rao bound on a particular parameter estimate [3] can be calculated as

$$
C_{\theta_{i}}=\left(F(\theta)^{-1}\right)_{i i}=\left(\left(\left[\begin{array}{c}
\frac{\partial \mathbf{x}_{j}^{T}}{\partial \mathbf{A}} \\
\frac{\partial \mathbf{x}_{j}^{T}}{\partial \mathbf{p}}
\end{array}\right]\left[\frac{\partial \mathbf{x}_{j}}{\partial \mathbf{A}} \frac{\partial \mathbf{x}_{j}}{\partial \mathbf{p}}\right]\right)^{-1}\right)_{i i}
$$

We recall that the Cramer-Rao bounds are lower bounds on the variance of any estimate of a parameter or parameter combination and as such indicate the estimability of the poles and residues.

Let us now examine the effect of the residue sizes on the Cramer-Rao bounds for each pole's estimate (i.e., on the diagonal entry of the Cramer-Rao bound matrix corresponding to the pole of interest). We first show that this Cramer-Rao bound is inversely proportional to the square of the corresponding residue.

Lemma 1

Consider two transfer functions $H(z)$ and $H^{*}(z)$ with the same pole locations but with residues $A_{0}, \ldots, A_{n-1}$ and $A_{0}^{*}, \ldots, A_{n-1}^{*}$. Let us consider the Cramer-Rao bounds on the estimation error variance for a pole $p_{i}$, when the impulse responses of $H(z)$ and $H^{*}(z)$ are used for estimation, respectively. The ratio of these variances is $\frac{\left(A_{i}^{*}\right)^{2}}{A_{i}^{2}}$.

Proof

The Fisher information matrix for the estimation problem when the impulse response of $H(z)$ is used can be written as 


$$
F(\theta)=\tilde{A} Q Q^{T} \tilde{A}
$$

where

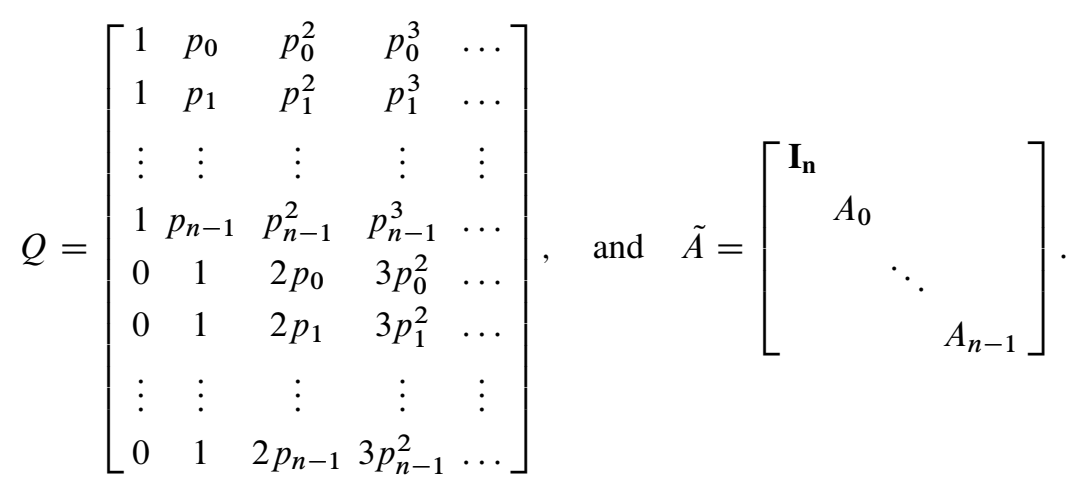

In this notation, the Cramer-Rao bounds are specified by the matrix

$$
C_{\theta}=\tilde{A}^{(-1)}\left(Q Q^{T}\right)^{-1} \tilde{A}^{-1}
$$

Exactly, analogous expressions hold when the impulse response of $H^{*}(z)$ is used but with $A_{i}$ replaced by $A_{i}^{*}$.

For each Cramer-Rao matrix, the bound on the estimation variance for the pole $p_{i}$ is given by the diagonal entry $n+i+1$ of the matrix. Noticing that the matrix $\left(Q Q^{T}\right)^{-1}$ depends solely on the (common) poles of the transfer functions $H(z)$ and $H^{*}(z)$ and hence is identical in the two cases, we immediately recover the result of the theorem.

The dependence of the Cramer-Rao bound on the pole locations is more intricate than the dependence on the residues. The Cramer-Rao bound is 0 (i.e., perfect estimation is possible) when the pole is on or outside the unit circle, such as the pole at unity in the diffusion model. The other diagonal entries are difficult to compute explicitly, but lower bounds can be developed by inverting principal submatrices of the Fisher information matrix, or equivalently by considering estimation assuming that only a subset of the parameters are unknown. Here, let us present two simple lower bounds, one that shows that poles near the origin tend to be harder to estimate than ones near \pm 1 and another that shows that two nearly-equal poles in a transfer function are hard to estimate. Here are the results.

\section{Lemma 2}

Consider the Cramer-Rao lower bound $C_{i}$ on the error variance for the estimate of a particular pole $p_{i}$, where $-1<p_{i}<1$. The following lower bound on $C_{i}$ holds: $C_{i} \geqslant \frac{\left(1-p_{i}^{2}\right)^{3}}{A_{i}^{2}\left(1+p_{i}^{2}\right)}$. This lower bound is approximately $\frac{1}{A_{i}^{2}}$ for $p_{i}$ near 0 and approximately $\frac{4\left(1-p_{i}\right)^{3}}{A_{i}^{2}}$ for $p_{i}$ near 1 .

Proof

With some algebra, we find that the diagonal entry of $F(\theta)$ corresponding to the estimate of the pole $p_{i}$ is $\frac{A_{i}^{2}\left(1+p_{i}^{2}\right)}{\left(1-p_{i}^{2}\right)^{3}}$. The corresponding diagonal entry of Cramer-Rao bound matrix is lower bounded by the inverse of this entry (which is a principal submatrix of $F(\theta)$, and the result is recovered.

\section{Lemma 3}

Consider a transfer function that has a pole $p_{0}$ (with $-1<p_{0}<1$ ) and another pole $p_{1}$ that is close to $p_{0}$, specifically $p_{1}=p_{0}+\delta$ (where $-1<p_{1}<1$ also) for small $\delta$. The Cramer-Rao bound on $p_{0}$ can be lower bounded by a function of $\delta$ that is on the order of $\frac{1}{\delta^{2}}$. 
Proof

The Cramer bound on $p_{0}$, denoted as $C_{p_{0}}$, satisfies $C_{p_{0}} \geq\left(\frac{\partial \mathbf{x}_{j}^{T}}{\partial \mathbf{p}} \frac{\partial \mathbf{x}_{j}}{\partial \mathbf{p}}\right)_{11}^{-1}=A_{0}^{-2}\left(P^{T} P\right)_{11}^{-1}$, where

$$
P^{T}=\left[\begin{array}{ccccc}
0 & 1 & 2 p_{0} & 3 p_{0}^{2} & \ldots \\
0 & 1 & 2 p_{1} & 3 p_{1}^{2} & \ldots \\
\vdots & \vdots & \vdots & \vdots & \vdots \\
0 & 1 & 2 p_{n-1} & 3 p_{n-1}^{2} & \cdots
\end{array}\right]
$$

Let us apply a transformation $\mathcal{L}$ so that $P$ is decomposed as $\mathcal{L} \hat{P}$, where $\hat{P}$ is same as $P$ except that the first row of $\hat{P}$ equals the difference between the first two rows of $P$. It is immediately clear that $\left(\hat{P}^{T} \hat{P}\right)_{11}$ is on the order of $\delta^{2}$. Simple calculation shows that $\left(\hat{P}^{T} \hat{P}\right)_{11}^{-1}$ and hence $\left(P^{T} P\right)_{11}^{-1}$ is on the order of $\frac{1}{\delta^{2}}$.

\subsection{Cramer-Rao bounds and pole-zero structure}

Several recent studies have shown the deep connection between a network's topological structure and the invariant-zero structure of certain system dynamics defined thereof ([21], see also [22] for background on invariant zeros). Thus, we are motivated to make explicit the relationship between the locations of the system zeros $(1,2)$ and the Cramer-Rao bound, as a step toward developing graph-theoretic characterizations. We make this connection in this subsection, by exploiting the relationship between the pole-zero and pole-residue forms.

The pole-zero form of the transfer function for the Laplacian network is

$$
H(z)=\frac{f(z)}{g(z)}=\frac{\gamma \prod_{i=1}^{m}\left(z-z_{i}\right)}{\prod_{i=0}^{n-1}\left(z-p_{i}\right)},
$$

where the real numbers $z_{i}, i=1, \ldots, m$ are the zeros of the system, $\gamma$ is a scaling constant, and the number of zeros $m$ is less than or equal to $n$ (strictly less if the stimulus and measurement are at different locations), and $f(z)$ and $g(z)$ are convenient notations for the numerator and denominator of $H(z)$. It is worth stressing that the poles of the system are purely a function of the underlying graph, while the number and locations of the zeros depend on the stimulus and measurement locations in addition to the graph.

The following lemma makes explicit the dependence of the Cramer-Rao bound on the zero locations.

\section{Lemma 4}

Consider two transfer functions $H_{1}(z)$ and $H_{2}(z)$ with the same pole locations (or denominator $g(z))$. Assume that the two transfer functions have different numerators $f_{1}(z)$ and $f_{2}(z)$. Consider the Cramer-Rao bounds on the estimation error variance for pole $p_{i}$, from the impulse responses of systems $H_{1}(z)$ and $H_{2}(z)$, respectively. The ratio of these two variances is $\frac{\left(f_{2}\left(p_{i}\right)\right)^{2}}{\left(f_{1}\left(p_{i}\right)\right)^{2}}$.

\section{Proof}

In the expression for the Fisher information matrix (6), the matrix $Q Q^{T}$ depends solely on the (common) poles of the transfer functions $H_{1}(z)$ and $H_{2}(z)$. To obtain a result in terms of poles and zeros, it only remains to express the residues $A_{0}, \ldots, A_{n-1}$ in terms of the poles and zeros. This can be performed using the classical partial fraction decomposition, which yields $A_{i}=\frac{f_{1}\left(p_{i}\right)}{\prod_{j \neq i}\left(p_{i}-p_{j}\right)}$ for the transfer function $H_{1}(z)$, and $A_{i}=\frac{f_{2}\left(p_{i}\right)}{\prod_{j \neq i}\left(p_{i}-p_{j}\right)}$ for the transfer $H_{2}(z)$. Substituting for the residues, the Cramer-Rao bound when the impulse response of the system $H_{1}(z)$ is used for estimation is 


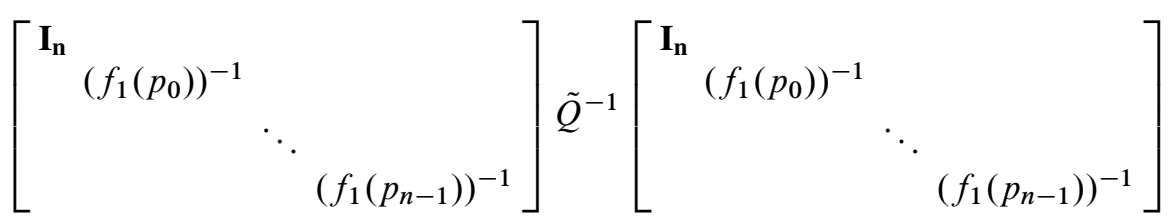

where $\tilde{Q}$ is purely a function of the pole locations. Similarly, the Cramer-Rao bound with the use of $H_{2}(z)$ is and

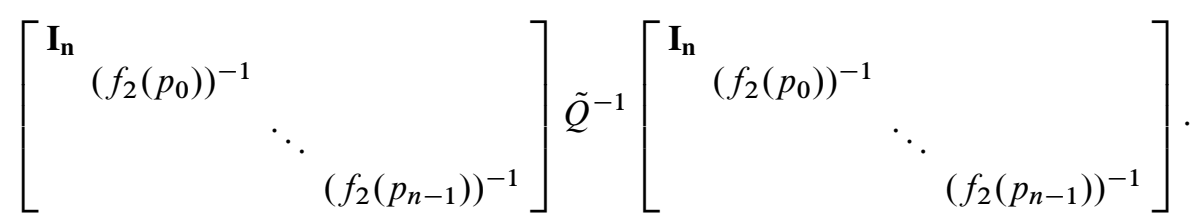

Comparing the diagonal entries of these matrices, we immediately recover the claim.

\section{Remark 1}

It follows from the aforementioned result that if there is a zero near a pole for a particular stimulusobservation pair, then the numerator of the transfer function evaluated at the pole is small, and hence, the estimability of the pole is poor.

\subsection{An eigenstructure-based characterization}

In this section, we translate the pole-residue and pole-zero-based results for the Cramer-Rao bounds into characterizations based on the spectrum $A$ of the network's state matrix. For the Laplacian network, this spectral characterization is useful as a stepping stone toward graph-theoretic results. Specifically, our aim here is to compare the Cramer-Rao bound for different stimulation and observation locations $i$ and $j$, by expressing the residues in terms of the spectrum of the Laplacian matrix and these locations. We obtain the following core result.

\section{Theorem 1}

Let us consider estimating a particular eigenvalue $\lambda_{r}$ of the Laplacian network (assuming that all the eigenvalues are nonrepeated), and let us denote the corresponding right eigenvector of the state matrix (normalized to unit length) by $\mathbf{v}_{r}$. Then, the estimation of the eigenvalue from a particular stimulation and observation pair $(i, j)$ is possible (i.e., the Cramer-Rao bound is finite) if and only if $v_{r i} v_{r j} \neq 0$. Furthermore, the Cramer-Rao bound on the eigenvalue estimate scales inversely with $\left(v_{r i} v_{r j}\right)^{2}$.

Proof

The impulse response of the Laplacian network with stimulus at component $i$ and measurement at component $j$ can be found using standard linear systems analysis, as $y[k]=\mathbf{e}_{j}^{T} V \Lambda^{k-1} V^{T} \mathbf{e}_{i}$, $k=1,2,3, \ldots$, where $\Lambda$ is a diagonal matrix containing the eigenvalues of the state matrix and $V$ is a matrix whose columns are the corresponding eigenvectors. Simplifying, we obtain that the impulse response is $y[k]=\sum_{r=0}^{n-1} v_{r i} v_{r j} \lambda_{r}^{k-1}$ for $k=1,2,3, \ldots$ Meanwhile, taking an inverse transform of the transfer function in the pole-residue form, we also can write the impulse response as $y[k]=\sum_{r=0}^{n-1} A_{r} \lambda_{r}^{k-1}$ for $k=1,2,3, \ldots$ Matching the two equations, we obtain that $A_{r}=v_{r i} v_{r j}$. Invoking (6), we recover the result of the theorem.

\section{Remark 2}

The equivalence used to prove the theorem also yields an interesting connection between the zeros of the transfer function and the eigenvector components of the state matrix (and hence the Laplacian matrix). In particular, the numerator of the transfer function evaluated at a particular pole $\left(f\left(\lambda_{r}\right)\right)$ equals the cross-participation factor $v_{r i} v_{r j}$. The participation-factor concept was originally used in power network applications to calculate the contribution of a system's modes to its dynamics at various network locations $[23,24]$ and in turn to facilitate mode computation and model aggregation. The aforementioned theorem shows that the participation factors also have significant 
value in evaluating identifiability of a system from measurement data and hence play important roles in sensor and actuation placement to achieve high-performance mode estimation from impulse-response data.

\subsection{Graph-theoretic bounds: the slow-coherency case}

We develop graph-theoretic results from the above-derived Cramer-Rao bounds, by drawing on the extensive algebraic-graph-theory literature [20] and on recent results connecting a Laplacian network's pole-zero structure with its graph [21]. In this section, we consider graphs with a special slow-coherency structure, that is, ones with tightly connected portions that are loosely connected in between. In such networks, which are often apt descriptions of infrastructures [18], typical disturbances propagate quickly locally while propagating slowly and in a synchronous fashion to the rest of the network. This special characteristic of the dynamics should critically impact the estimability of modal dynamics from different parts of the network. In this section, we verify that slow-coherency structures limit the performance of eigenvalue estimators. Specifically, we show that stimulation and measurement locations must be chosen carefully based on prior knowledge of slow-coherency structures, to achieve small Cramer-Rao bounds.

Let us begin with several preliminaries. Formally, let us consider a graph $\Gamma$ that has two tightly connected subgraphs $\left(\Gamma_{1}\right.$ and $\left.\Gamma_{2}\right)$ and weak links in between of order $\epsilon$ (where $\epsilon$ is much smaller than the edge weights in each subgraph). We note that the state matrix of the Laplacian network has the form $A=\left[\begin{array}{ll}A_{\Gamma_{1}} & \\ & A_{\Gamma_{2}}\end{array}\right]+\epsilon \widehat{A}$, where $A_{\Gamma_{1}}$ and $A_{\Gamma_{2}}$ are state matrices for the Laplacian networks defined by graphs $\Gamma_{1}$ and $\Gamma_{2}$, respectively, and the matrix $\epsilon \widehat{A}$ captures the interconnections between the two subgraphs. For small $\epsilon$, the matrix $A$ has one eigenvalue at 1 , and an eigenvalue that is within order- $\epsilon$ of 1 (termed the Fiedler eigenvalue). Each remaining eigenvalue of $A$ is a small perturbation $(O(\epsilon))$ of either a nonunity eigenvalue of $A_{\Gamma_{1}}$ or one of $A_{\Gamma_{2}}$; let us refer to each of these eigenvalues as being associated with either subgraph $\Gamma_{1}$ or subgraph $\Gamma_{2}$.

Now, consider the circumstance that both the stimulus and observation are placed at vertices in one subgraph, say $\Gamma_{1}$. Let us consider the transfer function $H_{\epsilon}(z)$ for this stimulus-observation pair, as a function of $\epsilon$ (including for the case that $\epsilon=0$ ). The following can be inferred about the transfer function $H_{\epsilon}(z)$ through simple system-theory and perturbation arguments:

(i) $H_{0}(z)$ can be written as $\frac{f_{0}(z)}{g_{0}(z)}$, where $g_{0}(z)$ is the characteristic polynomial of the Laplacian network with $\epsilon=0$ and $f_{0}(z)$ is a polynomial of degree less than $n$. Because the eigenvalues associated with $\Gamma_{2}$ are unobservable and uncontrollable for $\epsilon=0$, each such eigenvalue is also a root of the polynomial $f_{0}(z)$ - that is, the mode is canceled in the input-output dynamics.

(ii) For any particular $\epsilon, H_{\epsilon}(z)$ can be written as $\frac{f_{\epsilon}(z)}{g_{\epsilon}(z)}$, where $g_{\epsilon}(z)=g_{0}(z)+\widehat{g}(z)$ is the characteristic polynomial of $A$, and $\widehat{g}(z)$ is a polynomial that has coefficients of order $\epsilon$. Similarly, the numerator polynomial $f_{\epsilon}(z)$ can be written as $f_{0}(z)+\widehat{f}(z)$, where $\widehat{f}(z)$ is a polynomial of degree less than $n$ whose coefficients are of order $\epsilon$. We note from perturbation arguments that each root of $g_{\epsilon}(z)$ is a root of $g_{0}(z)$, perturbed by order $\epsilon$. Meanwhile, the numerator polynomial $f_{\epsilon}(z)$ may in general have different degree than $f_{0}(z)$. However, specifically, the degree of $f_{\epsilon}(z)$ will be at least as large as the degree of $f_{0}(z)$ for small enough $\epsilon$. Furthermore, it can easily be shown through perturbation arguments that $f_{\epsilon}(z)$ has one root close to each root of $f_{0}(z)$.

Using these observations and applying Lemma 4, we obtain the following.

\section{Theorem 2}

Consider a graph $\Gamma$ that has two tightly connected subgraphs $\left(\Gamma_{1}\right.$ and $\left.\Gamma_{2}\right)$ and weak links of order $\epsilon$. Assume that both stimulus and observation are placed at nodes in $\Gamma_{1}$. The Cramer-Rao bound on the estimate variance for an eigenvalue associated with $\Gamma_{2}$ is at least on the order of $\frac{1}{\epsilon^{2}}$. Meanwhile, the Cramer-Rao bound on the estimate variance for an eigenvalue associated with $\Gamma_{1}$ is within order $\epsilon$ of the Cramer-Rao bound that would be obtained if the estimate were performed from the Laplacian network defined solely from $\Gamma_{1}$ (with same stimulus and observation). 


\section{Proof}

First, let us consider the estimation of an eigenvalue associated with $\Gamma_{2}$. When all weak links are removed, all residues associated with $\Gamma_{2}$ are 0 . Reintroducing the weak links can only move these residues to an order of $\epsilon$ away from 0 . Hence, from Lemma 1, the Cramer-Rao bound on the estimate of a pole associated with $\Gamma_{2}$ is $O\left(\frac{1}{\epsilon^{2}}\right)$.

Now let us consider the case that both stimulus and observation are in $\Gamma_{1}$, and we try to estimate $p_{\epsilon i}$, the $i$ th pole associated with $\Gamma_{1}$, from the impulse response of $H_{\epsilon}(z)$. The introduction of the weak link only moves $p_{\epsilon i}$ from its original location $p_{o i}$ by $O(\epsilon)$. According to Lemma 4, the ratio of the Cramer-Rao bounds on pole $i$ 's estimate from the impulse responses of $H_{0}(z)$ and $H_{\epsilon}(z)$ are within $O(\epsilon)$ of $\frac{f_{\epsilon}\left(p_{\epsilon i}\right)}{f_{0}\left(p_{o i}\right)}$. The result follows from observation 2 presented earlier.

Thus, we see that stimulation and measurement within a strongly connected subgraph does not permit effective estimation of the eigenvalues associated with the other subgraph but can potentially be used for the estimation of local eigenvalues. We can also show that stimulation in one subgraph and measurement in the other subgraph does not permit accurate estimation of any eigenvalue.

\section{Theorem 3}

Consider a graph $\Gamma$ that has two tightly connected subgraphs $\left(\Gamma_{1}\right.$ and $\left.\Gamma_{2}\right)$ and weak links of order $\epsilon$. Assume that the stimulus location is associated with a vertex in $\Gamma_{1}$ and the observation location is associated with at a vertex in $\Gamma_{2}$. The Cramer-Rao bound on the estimate variance for an eigenvalue associated with either $\Gamma_{1}$ or $\Gamma_{2}$ is on the order of $\frac{1}{\epsilon^{2}}$.

\section{Proof}

When the weak links are removed, all residues in the pole-residue form are 0 , because the stimulus can not have any impact on the observation. The introduction of weak links can only perturb residues at an order of $\epsilon$ away from 0 , and hence the Cramer-Rao bounds on all pole estimates are at the order of $O\left(\frac{1}{\epsilon^{2}}\right)$.

In sum, we have shown that high-fidelity estimation of a network's modes requires stimulus and measurement within each strongly connected component within the network. These results can guide sensor/actuator placement (or experiment design) for mode estimation, when rough information about the network topology (e.g., presence/absence of coherency structures) is available.

\subsection{Graph-theoretic bounds: trees and more general graphs}

Here, we develop several relationships between a network's graph topology and Cramer-Rao bounds on mode estimates, for trees and general graphs (even when they do not have coherency structures). These graph-theoretic characterizations can inform stimulus and measurement selection for a strongly connected network or subnetwork.

We first present a simple result showing that exchanging the actuation and measurement locations does not change the Cramer-Rao bound on any pole.

\section{Lemma 5}

Consider the estimation of an eigenvalue $\lambda_{r}$ of the Laplacian network from impulse response data, where the actuation location is vertex $i$ and the measurement location is the distinct vertex $j$. The Cramer-Rao bound for $\lambda_{r}$ does not change if the actuation and measurement locations are switched, that is, if the actuation location is $j$ and the measurement location is $i$.

\section{Proof}

According to Theorem 1, the Cramer-Rao Bound (CRB) for $\lambda_{r}$ scales inversely with $\left(v_{r i} v_{r j}\right)^{2}$, when the actuation location is $i$ and the measurement location is $j$. Specifically, the CRB is equal to a quantity $\beta$ that depends only on the poles of the Laplacian network, scaled by $\left(v_{r i} v_{r j}\right)^{2}$. When the actuation and measurement locations are reserved, we see that the CRB equals $\beta$ scaled by $\left(v_{r j} v_{r i}\right)^{2}$. Hence, we immediately recover that the Cramer-Rao bound is unchanged.

The next theorem clarifies the role of the actuation and measurement locations on the CRB, when the network graph is a tree. Specifically, the theorem shows that many trees will have a central 
location such that estimation of the Fiedler eigenvalue is impossible if actuation or measurement is made at that location. The theorem also shows that stimulus and measurement corresponding to the leaves of the longest path in the tree yields the lowest CRB.

\section{Theorem 4}

Let us consider estimation of the Fiedler eigenvalue of the Laplacian network, when the graph is a tree. The minimum CRB is achieved when the measurement and actuation locations correspond to certain leaves in the tree. Moreover, the maximum Cramer-Rao bound is always achieved when the measurement and actuation location are among the vertices adjacent to the algebraic center of the tree. If the algebraic center is a vertex in the tree, inference is not possible if either the actuation or measurement location correspond to this vertex.

\section{Proof}

The Cramer-Rao bound on the Fiedler eigenvalue estimate scales inversely with $\left(v_{1 i} v_{1 j}\right)^{2}$, where $i$ is the actuation location, $j$ is the measurement location, and $v_{1}$ is the eigenvector associated with the Fiedler eigenvalue, commonly called the Fiedler eigenvector. According to [25, 26], entries in the Fiedler eigenvector increase or decrease monotonically from the algebraic center of the tree. As such, the maximum values of eigenvector entries are achieved at the leaves, and the minimum are among the nodes adjacent to the algebraic center of the tree. Moreover, if the algebraic center is a node (denoted as $k$ ) in a tree, the entry $v_{1 k}$ in the Fielder eigenvector is 0 according to Theorem 4.3 in [26]. In this case, we obtain that the CRB is infinite, indicating that estimating the Fiedler eigenvalue is impossible.

For tree graphs, the aforementioned theorem shows that the optimal estimation of the Fiedler eigenvalue is achieved (in the sense that the Cramer-Rao bound is minimized), using collocated actuation and observation at a leaf vertex far away from the center of the graph. For more general graphs, the Cramer-Rao bound also decreases when the actuation/observation locations are moved away from the graph center, but the pattern is more sophisticated. Here is the result.

\section{Theorem 5}

Consider a Laplacian network with connected graph $G$, and consider the estimation of the Fiedler eigenvalue from impulse-response data assuming a collocated actuation and observation location in the network. An edge cut of this graph that partitions the vertices into two connected subgraphs can be found, which has the following property; for every vertex, there is a path from the cut to the vertex such the Cramer-Rao bound is nonincreasing when the actuation/measurement location is moved along the path.

\section{Proof}

It is well-known, from the nodal-domain theory for Laplacian matrices, that any connected graph has an edge cut with the following properties: (i) the two partitions formed by the cut are connected, and (ii) the Fiedler eigenvector entries associated with one partition are greater than or equal to 0 , and the entries associated with the other partition are less than 0 . That is, the negative-valued and nonnegative-valued entries in the Fiedler eigenvector induce two connected subgraphs of the original graph. Let us verify that this cut satisfies the property claimed in the theorem. To do so, assume otherwise. Then there is a vertex in one of the two partitions, such that there is no path from the cut to the vertex such that the Cramer-Rao bound is nonincreasing when the actuation/measurement location is moved along the path. Let us consider the case that this vertex is in the partition with nonnegatively-valued Fiedler eigenvector components. If this is the case, however, there is at least one vertex in the partition (i) that is not adjacent to the cut and (ii) whose Fiedler eigenvector component is majorized by its neighbors and strictly majorized by at least one neighbor. However, from the eigenvector equation, we immediately find that the corresponding Fiedler eigenvector component would be negative, and thus, a contradiction is obtained. An analogous contradiction is obtained in the case that the vertex of interest is in the partition with negatively valued Fiedler eigenvector components. 
In sum, Theorem 5 shows that, for general graphs, Cramer-Rao bounds decrease, and hence, better estimation is possible along paths away from a central cut of the network.

The next result details the complex dependence of the CRB on the edge weight, for a simple graph with only two vertices. The result highlights essential tradeoffs in estimation performance as edge weights are strengthened or weakened in a graph.

\section{Theorem 6}

Consider a Laplacian network whose graph has two vertices. Assume that the actuation location is vertex 1 and the measurement location is vertex 2, and consider the CRB on the estimate of the Fiedler eigenvalue. Increasing the edge weight between the two nodes from 0 to 1 (the maximum stabilizing weight) causes the CRB to increase monotonically and then decrease monotonically. The largest CRB occurs when the edge weight is 0.5 .

\section{Proof}

In the two-vertex case considered here, the CRB on the Fiedler eigenvalue's estimate can be computed explicitly, as $\frac{\left(1-p_{1}^{2}\right)^{3}}{A_{1}^{2}\left(1+p_{1}^{2}\right)}$, where $A_{1}=\left(v_{11} v_{12}\right)$. Because the sum of the entries in the Fielder eigenvector equals 0 , we easily obtain that $v_{11}=-v_{12}=\sqrt{0.5}$ regardless of the edge weight between the vertices. Thus, $A_{1}$ is a constant, and the CRB is only dependent on the pole $p_{1}$. As the weight of the edge between the two vertices is increased from 0 to 1 , the Fielder eigenvalue $p_{1}$ decreases from 1 to -1 . It is thus clear that the CRB increases and then decreases, with the maximum corresponding $p_{1}=0$ or, equivalently, an edge weight of 0.5 .

Equations (7) and (8) indicate a special structure to the full Cramer-Rao bound matrix, which permit further structural/graphical characterization of the bound. Specifically, from these expressions, we see that the matrix $\tilde{Q}^{-1}$ is dependent solely on the pole locations and has no dependence on the actuation and observation locations. Meanwhile, the entries in the diagonal scaling matrices in (7) are either unity or depend on the actuation and observation location and more specifically are equal to modal participation factors associated with the actuation and observation locations. Let us draw on (7) and (8) to develop lower bounds on estimation performance, which are independent of the stimulus and observation location. Specifically, we study features of the Cramer-Rao bound matrix that characterize estimation performance, including its determinant, trace, and diagonal entries (see, e.g., [27] for concrete motivations for considering these features). The following theorem bounds these features, solely in terms of properties of the matrix $\tilde{Q}^{-1}$ and hence only in terms of the pole locations.

\section{Theorem 7}

Let $\alpha_{1}, \ldots, \alpha_{2 n}$ be the diagonal entries of $\tilde{Q}^{-1}$ (in order), and let $\beta_{1}, \ldots, \beta_{2 n}$ be the corresponding diagonal entries of the Cramer-Rao bound $C_{\theta}$. Then we have $\beta_{i} \geqslant \alpha_{i}$. Also, we have $\operatorname{tr}\left(C_{\theta}\right) \geqslant$ $\left(\sum_{i=1}^{n} \alpha_{i}+\sum_{i=n+1}^{2 n} \alpha_{i}^{1 / 3}\right)^{3} \geqslant \operatorname{tr}\left(\tilde{Q}^{-1}\right)$. Finally, we have that $\operatorname{det}\left(C_{\theta}\right) \geqslant n^{2 n} \operatorname{det}\left(\tilde{Q}^{-1}\right)$.

\section{Proof}

The diagonal entries of the Cramer-Rao bound corresponding to the residue estimates are equal to the corresponding diagonal entries of $\tilde{Q}^{-1}$, that is, $\alpha_{i}=\beta_{i}$ for $i=1, \ldots, n$. Meanwhile, the Cramer-Rao bound corresponding to each pole estimate involves a scaling of the associated diagonal entry of $\tilde{Q}^{-1}$ by a squared cross-participation factor. Thus, we can write each $\beta_{i}$, $i=n+1, \ldots, 2 n$ as $\beta_{i}=\frac{\alpha_{i}}{q_{i}^{2}}$, where $q_{i}$ is the absolute value of the appropriate modal participation factor or cross-participation factor. Because the $q_{i}$ are (absolute values of) products of eigenvector components, it follows immediately that $q_{i} \leq 1$, and hence the majorization of $\alpha_{i}$ by $\beta_{i}$ follows immediately. Because the Laplacian matrix is symmetric, it also follows immediately that $\sum_{i} q_{i}=1$ if the actuation and measurement are collocated, and $\sum_{i} q_{i} \leq 1$ otherwise. The bound on the trace of the CRB matrix can then be obtained by minimizing $\sum_{i=1}^{2 n} \beta_{i}=\sum_{i=1}^{n} \alpha_{i}+\sum_{i=n+1}^{2 n} \frac{\alpha_{i}}{q_{i}^{2}}$ with respect to $q_{i}$, subject to the constraint $\sum_{i} q_{i}=1$. This minimization problem with linear constraint can be solved using a Lagrange-multiplier argument. The details of the argument are 
straightforward (involving elementary calculus), so we omit them. Using the fact that the determinant operation distributes over matrix multiplication, we immediately obtain from (8) that $\operatorname{det}\left(C_{\theta}\right)=\frac{1}{\prod_{i=n+1}^{2 n} q_{i}} \operatorname{det}\left(\tilde{Q}^{-1}\right)$. The bound in the theorem statement follows immediately from the arithmetic mean-geometric mean (AM-GM) inequality.

\section{Remark 3}

The determinant bound has a subtlety that requires some further discussion. Recalling that the dominant pole and corresponding residue can be estimated perfectly, the determinant of the CramerRao bound equals zero in the limit of a long observation result; in this sense, the result in the theorem, although formally correct, is not very useful. Rather, the result should be interpreted as providing a bound when a finite observation horizon is considered, or alternately can be modified slightly to characterize the determinant of the Cramer-Rao submatrix excluding the dominant eigenvalue. To save space and reduce notation, we have not formalized these cases.

We note that the actuation/measurement-dependent diagonal scaling of $\tilde{Q}^{-1}$ to find the CramerRao Bound is specified by the participation factors associated with the actuation and measurement locations. This sole dependence on participation factors allows development of several simple results on actuator/sensor placement, as summarized in the following corollary.

\section{Corollary 1}

Consider the Cramer-Rao lower bound on a particular pole's estimate, that is, the diagonal entry of $C_{\theta}$ corresponding to that pole. The minimum possible value for the bound, over possible actuator and sensor locations, is always achieved when the actuator and sensor are collocated. However, any particular actuator/sensor pair cannot provide good estimates of all modes, in the sense that the diagonal entries of $\tilde{Q}^{-1}$ will be scaled on average by at least $n^{2}$ in calculating the diagonal entries of $C_{\theta}$.

The proof of the corollary is very similar to that of Theorem 7 , so we omit it.

\section{ILLUSTRATIVE EXAMPLES}

In this section, we use two examples to illustrate the structural results that we have obtained for network estimation.

\subsection{Example 1: connections to dynamical-network security}

Our study on estimation performance informs the study of security in sensor networks. One core benefit of sensor networks with distributed computational capabilities, as compared to centralized systems, is that each sensor only holds partial information about network measurements and hence has an increased degree of security. However, there is a concern that an adversary can ascertain the network topology, status information, or network dynamics from observing these localized sensor measurements/computations over time, even in the presence of noise [6, 27]. The results developed in this paper are promising for allowing characterization of distributed sensor network security, in terms of the network's topology.

To illustrate this connection, let us present a preliminary security analysis for a canonical distributed consensus-building or data-fusion algorithm for sensor networks. Specifically, we study a network of 5 sensor nodes belonging to two subgroups with a weak link in between (Figure 1). Each sensor node updates its own sensor value based upon its neighbors' values according to the following rule:

$$
x_{i}[k+1]=x_{i}[k]-\sum_{f o r \forall j, \text { s.t., } i, j \text { are neighbors }} L_{i j}\left(x_{i}[k]-x_{j}[k]\right),
$$

where $L_{i j}$ is a scaling factor, as indicated by the edge weight between nodes $i$ and $j$. This iterative updating rule will allow all sensor nodes to eventually reach consensus. For this small example, we can easily obtain the consensus-building dynamics as $\mathbf{x}[k+1]=$ 


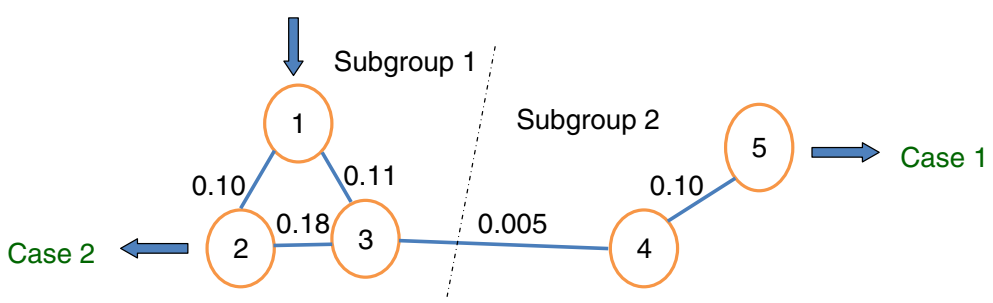

Figure 1. Topology of the 5-node distributed sensor network in Example 1.

$\left[\begin{array}{ccccc}0.79 & 0.10 & 0.11 & 0 & 0 \\ 0.10 & 0.72 & 0.18 & 0 & 0 \\ 0.11 & 0.18 & 0.705 & 0.005 & 0 \\ 0 & 0 & 0.005 & 0.895 & 0.10 \\ 0 & 0 & 0 & 0.10 & 0.90\end{array}\right] \mathbf{x}[k]$. Now let us say an adversary tries to infer network modes,

which capture the prominent features of network structure/dynamics. Suppose that an adversary pokes the system at location 1 with a unit impulse; that is, the adversary modifies the state or opinion or measurement of Sensor 1 from its nominal value (or knows of such modification), thus impacting the consensus-building dynamics. Let us compare the adversary's ability to estimate the modes corresponding to subgraph 1 , when measuring the response at node 5 (denoted as case 1) rather than at node 2 (denoted as case 2). We find that Case 2 permits a much higher fidelity estimate (or, equivalently, a much lower CRB). In fact, the CRB for case 2 is at a scaling of that for case 1 , by $0.005^{2}$ according to Theorem 3 . This conclusion is clear from the pole-residue form of the transform functions: $\frac{0.2}{z-1}+\frac{-0.2067}{z-0.99}+\frac{-0.0042}{z-0.68}+\frac{-0.0002}{z-0.53}+\frac{0.0110}{z-0.80}$ for case 1 and $\frac{0.2}{z-1}+\frac{0.1359}{z-0.99}+\frac{-0.3708}{z-0.68}+\frac{0.0348}{z-0.53}+\frac{0.0001}{z-0.80}$ for the case 2 . The two nonunity poles associated with subgroup 1 correspond to the third and fourth terms, and the nonunity pole associated with subgroup 2 corresponds to the fifth term. Clearly, the residues associated with the two poles in subgroup 1 are much smaller in case 1; this informs us that noise in the observation sequence can easily interrupt inference in this case. We find that the Cramer bounds for the two eigenvalues in subgraph 1 are $7.34 E 8 \sigma^{2}$ and $1.09 E 11 \sigma^{2}$ for the first case and $9.32 E 4 \sigma^{2}$ and $2.11 E 6 \sigma^{2}$ for the second case. Hence, to prevent estimation of the modes associated with a strongly connected subgraph, an adversary should be prevented from both actuating and observing the dynamics in that subgraph. Moreover, the Cramer-Rao bounds on the estimate of the pole associated with the second subgraph are $2.66 E 6 \sigma^{2}$ and $2.41 E 10 \sigma^{2}$ for cases 1 and 2 respectively, which are both quite large-that is, the adversary cannot estimate the modes associated with the second subgraph with high fidelity, in either configuration. Hence, the presence of slow-coherency structure can significantly enhance system security for sensor networks. However, we note that the slow-coherency structure also yields slowsettling consensus dynamics. Such tradeoffs between security and performance may be worthwhile to study in future work.

\subsection{Example 2: sensor/actuator placement for mode estimation}

The analyses developed here also facilitate sensor and actuator placement for mode estimation. In particular, (7) and (8) indicate that the cross-participation factors of the Lacan be used to compare estimation performance at multiple sensor locations. Specifically, if estimation of a particular mode $i$ is of importance, the Cramer-Rao bound on the mode estimate for different actuationmeasurement pairs $(j, k)$ is inversely proportional to the cross-participation-factor $v_{i j} v_{i k}$. Similarly, if the determinant of the Cramer-Rao bound is considered as an aggregate measure of estimation performance, then different actuation-measurement pairs $(j, k)$ can be compared based on products of the cross-participation factors, $\prod_{i} v_{i j} v_{i k}$; the determinant is inversely proportional to the inverse of this product. Because the spectra of Laplacian matrices are relatively easy to compute even when the dimension of the matrix is moderate or large, these relationships facilitate computation of good sensing locations. They also are a step toward graphical insights into sensor placement (e.g., as given in Theorem 5). 

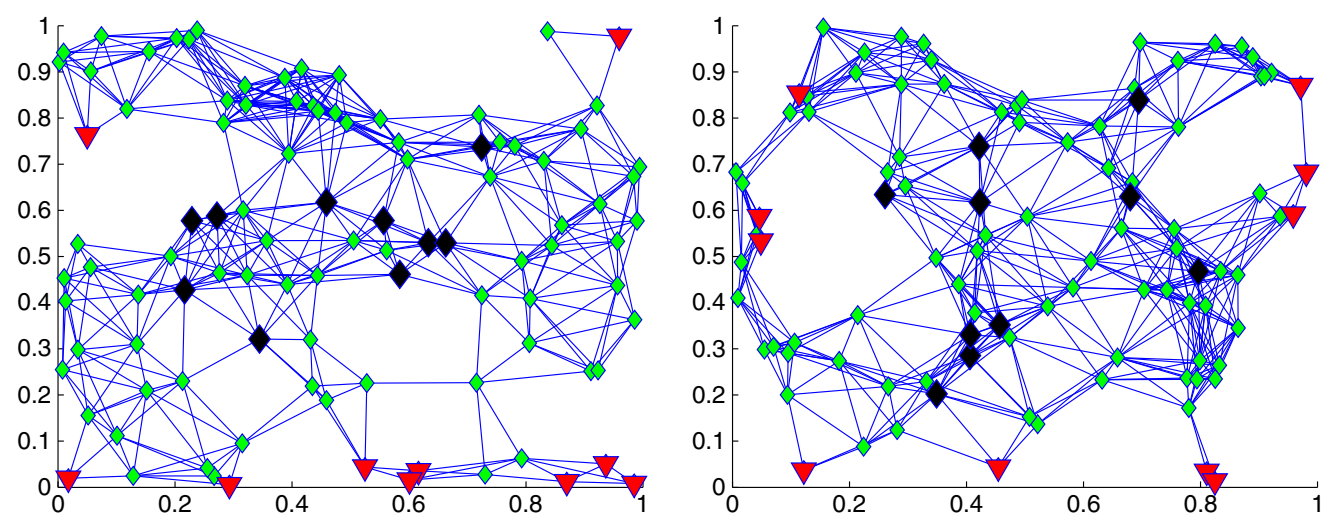

Figure 2. The 10 best and 10 worst locations for estimation are shown in two randomly constructed networks with 100 components. The best locations are labeled with black diamonds, and the worst with red triangles.

Here, we consider sensor placement to optimize the Cramer-Rao matrix determinant, in constructed networks with 100 components. For our example, network graphs are generated as follows: 100 vertices are located randomly (uniformly) in the unit square, and an edge is drawn between two vertices if the distance between them is sufficiently small; the edge weight is chosen uniformly on $[0,1]$. We consider mode estimation from impulse-response data, in the case that the actuation and measurement are collocated. Specifically, mode-estimation performance, as measured by the Cramer-Rao matrix determinant, is compared for actuator and sensor placement at each network component (using the participation-factor-based expression). In Figure 2, we illustrate the 10 most effective actuation/sensing locations and the 10 least effective locations, for four such constructed networks (generated as described earlier). The examples illustrate that effective actuation/sensing locations for mode estimation are typically highly-connected network components near the center of the network, while components at the periphery are ineffective for mode estimation. It is interesting that the Fiedler eigenvalue is effectively estimated from such peripheral nodes (Theorem 5), but overall mode-estimation performance (as measured by the Cramer-Rao matrix determinant) is optimized by centrally located sensors.

\section{FUTURE DIRECTIONS}

We have related a dynamical network's structure to the Cramer-Rao bound of mode estimates and have use that relation to guide the design of stimulus and measurement locations for network identification. The result shows the significance of coherency structures in the inference of network parameters. Beyond the coherency-focused results, we have shown that the zero structure of the input-output dynamics, or alternately the participation factors, generally plays an important role in determining mode estimability for particular stimulation and measurement locations. We have obtained some graph-theoretic characterizations of the participation factors and hence the CRB for certain graph classes and particularly for the Fiedler eigenvalue. We posit that these characterizations can be exploited to guide the design of stimulation/observation locations for graphs without coherency structures or within one strongly connected subgraphs. We will pursue such design and also seek for statistical characterization of mode estimation performance for classes of random graphs.

\section{ACKNOWLEDGEMENTS}

The first two authors contributed equally to this work. This research was partially supported by Wan's United States National Science Foundation (US NSF) grants CNS-1035386 and CNS-1058110 and Roy's US NSF grants ECS-0725589, ECS-0901137, and CNS-1058124. 


\section{REFERENCES}

1. In Blind Deconvolution, Haykin S (ed.). Prentice Hall: Upper Saddle River NJ, 1994.

2. Ljung L. System Identification-Theory for the User. Prentice Hall: Englewood Cliffs NJ, 1987.

3. Scharf LL. Statistical Signal Processing: Detection, Estimation, and Time-Series Analysis. Addison-Wesley: Reading MA, 1991.

4. Dunlop M, Murray R. Toward biological system identification: fast and accurate estimates of parameters in genetic regulatory networks. Proceedings of the 2006 Conference on Decision and Control, San Diego, CA, 2006.

5. Smith V, Yu J, Smulders T, Hartemink A, Jarvis E. Computational inference of neural information flow networks. PLoS Computational Biology 1996; 2:1436-1449.

6. Xue M, Roy S, Wan Y, Das S. Security and vulnerability of cyber-physical infrastructure networks: a controltheoretic approach. In Handbook on Securing Cyber-Physical Critical Infrastructure: Morgan Kaufmann, Waltham, MA, 2012; 5-30.

7. Roy S, Xue M, Das S. Security and discovery of spread dynamics in cyber-physical networks. IEEE Transactions on Parallel and Distributed Systems (Special Issue on Cyber Physical Systems) 2012; 23(9):1694-1707.

8. Wan Y, Roy S. On inference of network time constants from impulse response data: graph-theoretic cramer bounds. Proceedings of 48th IEEE Conference on Decision and Control, Shanghai, P.R. China, 2009; 4111-4116.

9. McWhorter LT, Scharf LL. Cramer-Rao bounds for deterministic modal analysis. IEEE Transactions on Signal Processing, 1993; 41(5):1847-1862.

10. Olfati-Saber R, Fax JA, Murray RM. Consensus and cooperation in networked multi-agent systems. Proceedings of IEEE 2007; 95(1):215-233.

11. Olfati-Saber R. Flocking for multi-agent dynamic systems: algorithms and theory. IEEE Transactions on Automatic Control 2006; 51(3):401-420.

12. Wan Y, Numuduri K, Akula S, Varanasi M. The impact of multi-group multi-layer network structure on the performance of distributed consensus building strategies. International Journal of Robust and Nonlinear Control 2013; 23:653-662.

13. Wan Y, Roy S. A scalable methodology for evaluating and designing coordinated air traffic flow management strategies under uncertainty. IEEE Transactions on Intelligent Transportation Systems 2008; 9(4):644-656.

14. Dosiek L, Trudnowski DJ, Pierre JW. New algorithms for mode shape estimation using measured data. Proceedings of the IEEE Power Engineering Society General Meeting, Pittsburgh, Pennsylvania, 2008.

15. Zhou N, Pierre JW, Trudnowski DJ, Guttromson RT. Robust RLS methos for online estimation of power system electromechanical modes. IEEE Transactions on Power Systems 2007; 22(3):1240-1249.

16. Chakrabortty A, Martin CF. Optimal measurement allocation for parametric model identification of electrical networks. Nonlinear Theory and its Applications, IEICE 2011; 2(3):302-319.

17. Sandberg H, Texiera A, Johansson KH. On security indices for state estimators in power networks. First Workshop on Secure Control Systems, Stockholm, Sweden, 2010.

18. Chow J (ed). Time-Scale Modeling of Dynamic Networks with Application to Power Systems. Springer-Verlag: Berlin, 1982.

19. Wu CW, Chua L. Application of kronecker products to the analysis of systems with uniform linear coupling. IEEE Transactions on Circuits and Systems I 1995; 42(10):775-779.

20. Chung FRK. Spectral Graph Theory. American Mathematical Society Press: Providence, RI, 1997.

21. Roy S, Wan Y, Saberi A. On time-scale designs for networks. International Journal of Control 2009; 82(7): 1313-1325.

22. Sannuti P, Saberi A. A special coordinate basis of multivariable linear systems, finite and infinite zero structure, squaring-down and decoupling. International Journal of Control 1987; 45(5):1655-1704.

23. Perez-Arriaga IJ, Verghese GC, Schweppe FC. Selective modal analysis with applications to electric power systems, Part I: Heuristic introduction. IEEE Transactions on Power Apparatus and Systems 1982; 101:3117-3125.

24. Verghese GC, Perez-Arriaga IJ, Schweppe FC. Selective modal analysis with applications to electric power systems, Part II: The dynamic stability problem. IEEE Transactions on Power Apparatus and Systems 1982; 101:3126-3134.

25. Fiedler M. Eigenvectors of acyclic matrices. Czechoslovak Mathematics Journal 1975; 25(100):607-618.

26. Fiedler M. Absolute algebraic connectivity of trees. Linear and Multilinear Algebra 1990; 26:85-106.

27. Rai A, Xue M, Yeung E, Roy S, Wan Y, Saberi A, Warnick S. Initial-condition estimation in network synchronization processes: graphical characterizations of estimator structure and performance. AIAA Guidance, Navigation, and Control Conference, August, Oregon, 2011. 